International Mathematical Forum, Vol. 9, 2014, no. 30, 1441 - 1446 HIKARI Ltd, www.m-hikari.com

http://dx.doi.org/10.12988/imf.2014.48152

\title{
Explicit Polynomial for Sums of Powers of Odd Integers
}

\author{
José Luis Cereceda
}

Distrito Telefónica, Edificio Este 1

28050 - Madrid, Spain

Copyright (c) 2014 José Luis Cereceda. This is an open access article distributed under the Creative Commons Attribution License, which permits unrestricted use, distribution, and reproduction in any medium, provided the original work is properly cited.

\begin{abstract}
In this note we derive the explicit, non-recursive form of the coefficients of the polynomial associated with the sums of powers of the first $n$ odd integers. As a by-product, we deduce a couple of new identities involving the Bernoulli numbers.
\end{abstract}

Mathematics Subject Classification: 11B57, 11C08, 11B68

Keywords: sums of powers, odd integers, polynomial representation, Bernoulli numbers

\section{Introduction}

In this note we deal with the sums of powers of the first $n$ odd integers

$$
O_{r}(n)=1^{r}+3^{r}+5^{r}+\cdots+(2 n-1)^{r},
$$

where $r=1,2,3, \ldots$. As we can learn from Theorem $\mathrm{V}$ in a paper by Witmer [4], it turns out that, for odd $r=2 p-1(p=1,2,3, \ldots), O_{2 p-1}(n)$ can be expressed as the even polynomial in $n$ :

$$
O_{2 p-1}(n)=\sum_{k=1}^{p} E_{p, k} n^{2 k}
$$


where the coefficients $E_{p, k}$ are rational numbers independent of $n$ that satisfy $E_{p, p}=2^{2 p-2} / p$, and the recursion formula

$$
E_{p, k}=-\frac{1}{2 p} \sum_{j=1}^{p-1}\left(\begin{array}{c}
2 p \\
2 j-1
\end{array}\right) E_{j, k}, \quad k<p .
$$

Note that, for the case $p=1$, from (1) we retrieve the well-known identity

$$
1+3+5+\cdots+(2 n-1)=n^{2} .
$$

On the other hand, from Theorem VI in [4], it follows that, for even $r=2 p$ $(p=1,2,3, \ldots), O_{2 p}(n)$ can be expressed as the odd polynomial in $n$ :

$$
O_{2 p}(n)=\sum_{k=0}^{p} F_{p, k} n^{2 k+1}
$$

where the coefficients $F_{p, k}$ are rational numbers independent of $n$ that satisfy $F_{p, p}=2^{2 p} /(2 p+1)$, and the recursion formula

$$
F_{p, k}=-\frac{1}{2 p+1} \sum_{j=0}^{p-1}\left(\begin{array}{c}
2 p+1 \\
2 j
\end{array}\right) F_{j, k}, \quad k<p .
$$

Moreover, Guo and Shen [2] gave recently the following factorized forms for $O_{2 p-1}(n)$ and $O_{2 p}(n)$ :

$$
\begin{aligned}
& O_{2 p-1}(n)=n^{2} \sum_{k=1}^{p} C_{p, k} n^{2 p-2 k} \\
& O_{2 p}(n)=n(2 n-1)(2 n+1) \sum_{k=1}^{p} D_{p, k}(2 n-1)^{p-k}(2 n+1)^{p-k}
\end{aligned}
$$

together with the corresponding recurrence formulae to compute the rational coefficients $C_{p, k}$ and $D_{p, k}(k=1,2, \ldots, p)$.

However, as far as the present author is aware, there exist no explicit (recurrence-free) formulae for either $E_{p, k}, F_{p, k}, C_{p, k}$, or $D_{p, k}$ in the current literature. The purpose of this note is to provide an explicit formula for these coefficients.

\section{Determination of the coefficients $E_{p, k}$ and $F_{p, k}$}

To obtain $E_{p, k}$, we employ, on the one hand, the easily confirmed relation

$$
O_{p}(n)=S_{p}(2 n)-2^{p} S_{p}(n)
$$


where $S_{p}(n)=1^{p}+2^{p}+3^{p}+\cdots+n^{p}$ is the ordinary power sum of the first $n$ positive integers. On the other hand, we use the well-known Bernoulli formula according to which (e.g. see [3])

$$
S_{p}(n)=\frac{1}{p+1} \sum_{i=0}^{p}\left(\begin{array}{c}
p+1 \\
i
\end{array}\right) B_{i} n^{p+1-i}
$$

where the $B_{i}$ 's are the Bernoulli numbers, namely $B_{0}=1, B_{1}=\frac{1}{2}, B_{2}=\frac{1}{6}$, $B_{3}=0, B_{4}=-\frac{1}{30}$, etc. Thus, using (5) and (6), we have

$$
\begin{aligned}
O_{2 p-1}(n) & =\frac{1}{2 p} \sum_{i=0}^{2 p-1}\left(\begin{array}{c}
2 p \\
i
\end{array}\right)\left(2^{2 p-i}-2^{2 p-1}\right) B_{i} n^{2 p-i} \\
& =\frac{1}{2 p} \sum_{j=1}^{2 p}\left(\begin{array}{c}
2 p \\
j
\end{array}\right)\left(2^{j}-2^{2 p-1}\right) B_{2 p-j} n^{j} \\
& =\frac{2^{2 p-2}}{p} n^{2 p}+\frac{1}{2 p} \sum_{j=1}^{2 p-2}\left(\begin{array}{c}
2 p \\
j
\end{array}\right)\left(2^{j}-2^{2 p-1}\right) B_{2 p-j} n^{j} .
\end{aligned}
$$

Since $B_{i}=0$ for all odd $i \geq 3$, this can be written as

$$
O_{2 p-1}(n)=\frac{1}{2 p} \sum_{k=1}^{p}\left(\begin{array}{l}
2 p \\
2 k
\end{array}\right)\left(2^{2 k}-2^{2 p-1}\right) B_{2 p-2 k} n^{2 k}
$$

Comparing this expression with (1), we deduce that

$$
E_{p, k}=\frac{1}{2 p}\left(\begin{array}{c}
2 p \\
2 k
\end{array}\right)\left(2^{2 k}-2^{2 p-1}\right) B_{2 p-2 k}, \quad k=1,2, \ldots, p .
$$

Proceeding the same way with $O_{2 p}(n)$, we get

$$
O_{2 p}(n)=\frac{1}{2 p+1} \sum_{k=0}^{p}\left(\begin{array}{l}
2 p+1 \\
2 k+1
\end{array}\right)\left(2^{2 k+1}-2^{2 p}\right) B_{2 p-2 k} n^{2 k+1},
$$

and then, from (2), we deduce that

$$
F_{p, k}=\frac{1}{2 p+1}\left(\begin{array}{c}
2 p+1 \\
2 k+1
\end{array}\right)\left(2^{2 k+1}-2^{2 p}\right) B_{2 p-2 k}, \quad k=0,1,2, \ldots, p .
$$

Let us note the relationships

$$
\left\{\begin{array}{l}
F_{p, 0}=\left(2-2^{2 p}\right) B_{2 p} \\
F_{p, k}=\frac{4 p}{2 k+1} E_{p, k}, \text { for } k=1,2, \ldots, p .
\end{array}\right.
$$


Furthermore, since $O_{2 p-1}(1)=O_{2 p}(1)=1$, we have that $\sum_{k=1}^{p} E_{p, k}=\sum_{k=0}^{p} F_{p, k}=$ 1 , from which we obtain the identities

$$
\sum_{k=1}^{p}\left(\begin{array}{c}
2 p \\
2 k
\end{array}\right)\left(2^{2 k}-2^{2 p-1}\right) B_{2 p-2 k}=2 p
$$

and

$$
\sum_{k=0}^{p}\left(\begin{array}{c}
2 p+1 \\
2 k+1
\end{array}\right)\left(2^{2 k+1}-2^{2 p}\right) B_{2 p-2 k}=2 p+1
$$

\section{Determination of the coefficients $C_{p, k}$ and $D_{p, k}$}

In view of equations (3) and (1), it follows that the coefficients $C_{p, k}$ and $E_{p, k}$ are related by $C_{p, k}=E_{p, p+1-k}(k=1,2, \ldots, p)$. Thus from $(7)$ we get

$$
C_{p, k}=\frac{2^{2 p-2}}{p}\left(\begin{array}{c}
2 p \\
2 k-2
\end{array}\right)\left(2^{3-2 k}-1\right) B_{2 k-2}, \quad k=1,2, \ldots, p .
$$

On the other hand, in order to determine the coefficients $D_{p, k}$, we rely on the following two pieces of information:

1. By Corollary 2.2 in [2], it holds that $D_{p, k}=2^{2 k-1} B_{p, k}(k=1,2, \ldots, p)$, where the $B_{p, k}$ 's are the (unspecified) coefficients of the polynomial for $S_{2 p}(n)$ :

$$
S_{2 p}(n)=2(2 n+1) S_{1}(n) \sum_{k=1}^{p} B_{p, k}\left(2 S_{1}(n)\right)^{p-k}
$$

where $S_{1}(n)=n(n+1) / 2$.

2. In [1], $S_{2 p}(n)$ is expressed as

$$
S_{2 p}(n)=\left(n+\frac{1}{2}\right) S_{1}(n) \sum_{j=0}^{p-1} F_{j}^{(2 p)}\left(S_{1}(n)\right)^{j}
$$

where $^{1}$

$$
F_{j}^{(2 p)}=8^{j+1} \sum_{m=1}^{p}\left(\begin{array}{c}
2 p \\
2 m
\end{array}\right)\left(\begin{array}{c}
m \\
j+1
\end{array}\right) \frac{2^{2 m+1-2 p}-1}{2^{2 m}(2 m+1)} B_{2 p-2 m} .
$$

\footnotetext{
${ }^{1}$ Strictly speaking, in [1] the summation appearing in the formula for $F_{j}^{(2 p)}$ starts at $m=0$. However, nothing changes if the summation starts at $m=1$ because the term corresponding to $m=0$ does vanish for all values of $j=0,1,2, \ldots, p-1$.
} 
Therefore, by equating the polynomials in (9) and (10), it is readily seen that $B_{p, k}=F_{p-k}^{(2 p)} / 2^{p+2-k}$, and then from (11) we obtain

$$
B_{p, k}=\frac{1}{2^{2 k-1}} \sum_{m=1}^{p}\left(\begin{array}{c}
2 p \\
2 m
\end{array}\right)\left(\begin{array}{c}
m \\
p+1-k
\end{array}\right) \frac{2-2^{2 p-2 m}}{2 m+1} B_{2 p-2 m} .
$$

To get the desired coefficients $D_{p, k}$, we simply have to multiply this expression by $2^{2 k-1}$ :

$$
D_{p, k}=\sum_{m=1}^{p}\left(\begin{array}{c}
2 p \\
2 m
\end{array}\right)\left(\begin{array}{c}
m \\
p+1-k
\end{array}\right) \frac{2-2^{2 p-2 m}}{2 m+1} B_{2 p-2 m}, \quad k=1,2, \ldots, p .
$$

From formula (12) we quickly find that $D_{p, 1}=1 /(2 p+1)$, a result already given in [2].

However, by using (12) we can directly evaluate the factorized form (4) of $O_{2 p}(n)$ without resorting to any recurrence formulae. As a simple example, next we give explicitly the polynomial for $O_{14}(n)$ whose coefficients are obtained from (12):

$$
\begin{aligned}
& 1^{14}+3^{14}+5^{14}+\cdots+(2 n-1)^{14} \\
= & n N\left(\frac{1}{15} N^{6}-\frac{28}{15} N^{5}+\frac{448}{15} N^{4}-\frac{2816}{9} N^{3}+\frac{91904}{45} N^{2}-7168 N+\frac{28672}{3}\right),
\end{aligned}
$$

where $N$ is shorthand notation for $(2 n-1)(2 n+1)$. Note that the obtained coefficients $D_{7, k}, k=1,2, \ldots, 7$, alternate in sign in accordance with the general rule $\operatorname{sgn} D_{p, k}=(-1)^{k-1}, k=1,2, \ldots, p$, conjectured in [2].

Further, we observe that $O_{2 p}(n)$ can alternatively be expressed as

$$
O_{2 p}(n)=n \sum_{k=1}^{p} D_{p, k}^{\prime} N^{k}
$$

where

$$
D_{p, k}^{\prime}=\sum_{m=1}^{p}\left(\begin{array}{c}
2 p \\
2 m
\end{array}\right)\left(\begin{array}{c}
m \\
k
\end{array}\right) \frac{2-2^{2 p-2 m}}{2 m+1} B_{2 p-2 m}, k=1,2, \ldots, p .
$$

Finally, we note the relationships between the coefficients $F_{p, k}$ of the polynomial (2) and the coefficients $D_{p, k}^{\prime}$ :

$$
\left\{\begin{array}{l}
F_{p, 0}=\sum_{k=1}^{p}(-1)^{k} D_{p, k}^{\prime} \\
F_{p, k}=2^{2 k} \sum_{s=k}^{p}(-1)^{s-k}\left(\begin{array}{l}
s \\
k
\end{array}\right) D_{p, s}^{\prime}, \text { for } k=1,2, \ldots, p
\end{array}\right.
$$


From the above relationships, we can deduce a couple of new identities involving the Bernoulli numbers. Indeed, using (13), and recalling that $F_{p, 0}=$ $\left(2-2^{2 p}\right) B_{2 p}$, from the first relationship we obtain

$$
\sum_{k=1}^{p}(-1)^{k} \sum_{m=1}^{p}\left(\begin{array}{c}
2 p \\
2 m
\end{array}\right)\left(\begin{array}{c}
m \\
k
\end{array}\right) \frac{2-2^{2 p-2 m}}{2 m+1} B_{2 p-2 m}=\left(2-2^{2 p}\right) B_{2 p} .
$$

Likewise, using (8) and (13), from the second relationship we obtain

$$
\begin{aligned}
\sum_{s=k}^{p}(-1)^{s-k}\left(\begin{array}{l}
s \\
k
\end{array}\right) \sum_{m=1}^{p}\left(\begin{array}{c}
2 p \\
2 m
\end{array}\right)\left(\begin{array}{c}
m \\
s
\end{array}\right) \frac{2-2^{2 p-2 m}}{2 m+1} B_{2 p-2 m} \\
=\frac{1}{2 p+1}\left(\begin{array}{c}
2 p+1 \\
2 k+1
\end{array}\right)\left(2-2^{2 p-2 k}\right) B_{2 p-2 k}
\end{aligned}
$$

which holds for $k=1,2, \ldots, p$.

\section{References}

[1] J.L. Cereceda, Explicit form of the Faulhaber polynomials, to appear in The College Mathematics Journal.

[2] S. Guo and Y. Shen, On sums of powers of odd integers, Journal of Mathematical Research with Applications 33 (2013), 666-672.

[3] H. Sherwood, Sums of power of integers and Bernoulli numbers, The Mathematical Gazette 54 (1970), 272-274.

[4] E.E. Witmer, The sums of powers of integers, The American Mathematical Monthly 42 (1935), 540-548.

\section{Received: September 3, 2014}

